\title{
A PROBLEM IN THE CALCULUS OF VARIATIONS WITH AN INFINITE NUMBER OF AUXILIARY CONDITIONS*
}

\author{
BY \\ R. G. D. RICHARDSON
}

INTRODUCTION

The significance of the calculus of variations as a focal point of analysis has been emphasized by Hilbert and his school, and its intimate connection with the theories of mechanics, differential equations, integral equations, and quadratic forms in an infinite number of variables, has been used to the mutual benefit of all these disciplines. From one standpoint the problems of the calculus of variations may be regarded as problems of ordinary maxima and minima in a denumerable or non-denumerable infinity of independent variables; the imposition of a finite number of auxiliary conditions would then be equivalent to reducing the infinity of variables by a finite number. It is natural to inquire what will happen when a denumerable infinity of auxiliary conditions are imposed on the function involved in the integral to be minimized. In various branches of mathematics much light has been thrown on problems by a generalization from the finite to the infinite and it may reasonably be expected that there will be additional insight into the problems of the calculus of variations by the development of a similar extension.

This paper undertakes to make a beginning of such a study by treating a particular problem which has for its Euler condition a differential equation central in mathematical physics. Some of the results will appear as natural generalizations of criteria already known, while others seem in contradiction to them.

The problem to be studied is intimately related to one discussed earlier $\dagger$ by the author in which a finite number of auxiliary conditions were imposed. That discussion concerned the solutions of the equation

$$
L(u) \equiv\left(p(x) u^{\prime}(x)\right)^{\prime}+q(x) u(x)+\lambda k(x) u(x)=0, p>0,
$$

subject to the boundary conditions

\footnotetext{
* Presented to the Society, September 11, 1925; received by the editors July 14, 1926.

$\dagger$ Das Jacobische Kriterium der Variationsrechnung und die Oszillationseigenschaften linearer Differentialgleichungen 2. Ordnung, Mathematische Annalen, vol. 68, p. 279.
} 


$$
u(0)=u(1)=0 \text {. }
$$

There are three distinct cases of the equation (0.1) which may be distinguished as follows:

(i). Orthogonal case. When $k(x)$ is of one sign; for example, $k(x)$ is positive or zero and equal to zero only at a finite number of points in the interval. The system (0.1), (0.2) has an infinite number of normalized characteristic solutions $U_{1}, U_{2}, \cdots$, corresponding to the characteristic numbers $\lambda_{1}<\lambda_{2}<\cdots$.

(ii). Polar case. When $k(x)$ has both signs and $q(x) \leqq 0$. The system (0.1), (0.2) has an infinite number of normalized characteristic solutions $U_{1}, U_{2}, \ldots$ corresponding to the positive characteristic numbers $\lambda_{1}<\lambda_{2}<\cdots$ and an infinite number $U_{-1}, U_{-2}, \cdots$ corresponding to the negative characteristic numbers $\lambda_{-1}>\lambda_{-2}>\cdots$.

(iii). Complex case.* When $k(x)$ has both signs and $q(x)$ is positive in at least part of the interval. The system $(0.1),(0.2)$ as in the polar case has two infinite sets of characteristic solutions and characteristic numbers. But, if $q(x)$ is large enough and positive, a finite number of the characteristic numbers $\lambda_{1}, \cdots, \lambda_{m_{1}}, \lambda_{-1}, \cdots, \lambda_{-m_{2}}$ are complex, as are also the characteristic solutions.

Exact theorems concerning the existence of extrema in the various cases are given in $\$ 3$. In the other sections, however, unless explicit mention is made to the contrary the discussion concerns only the orthogonal case. The argument can generally be carried over to the polar case as is occasionally indicated in the text or a footnote. In the complex case, the problems of the calculus of variations would ordinarily have no meaning.

Intimately related to the differential equation is the calculus of varia tions problem

$$
D(u) \equiv \int_{0}^{1}\left[p u^{\prime 2}-q u^{2}\right] d x=\min .
$$

the minimizing function $u(x)$ being subject to the boundary conditions $(0.2)$, the quadratic condition

$$
K_{0} \equiv \int_{0}^{1} k u^{2} d x=1
$$

and the linear conditions

\footnotetext{
* This case was treated by the author, Contributions to the study of oscillation properties of the solutions of linear differential equations of the second order, American Journal of Mathematics, vol. 40 (1918), p. 283.
} 


$$
K_{i} \equiv \int_{0}^{1} k U_{i} u d x=0 \quad(i=1, \cdots, m-1),
$$

where $U_{i}(x)$ denotes the solution of the corresponding extremum problem with $i-1$ linear conditions and which may be identified with the solutions $U_{i}(x)$ of $(0.1)$. The solution of the problem $(0.3),(0.2),(0.4),(0.5)$ is then furnished by $U_{m}(x)$ satisfying for $\lambda=\lambda_{m}$ the equation (0.1), to which the Euler condition of all the minimum problems for $m=1,2, \cdots$ may be reduced. From the equation

$$
\int_{0}^{1}\left(p U_{m}^{\prime 2}-q U_{m}^{2}\right) d x=\lambda_{m} \int_{0}^{1} k U_{m}^{2} d x,
$$

easily derived from (0.1), it will be noted that the value given to $D(u)$ by $U_{m}$ is $\lambda_{m}$.

The Legendre condition

$$
H_{y^{\prime} y^{\prime}}=2 p>0
$$

built up after the usual Lagrange method for the function $B \equiv p u^{\prime 2}-q u^{2}$ $+\lambda k u^{2}+\sum_{i=1}^{m-1} 2 \mu_{i} k U_{i}$, and the Weierstrass condition

$$
E \equiv p\left(u^{\prime}-p\right)^{2} \geqq 0
$$

are satisfied not only by $U_{m}$ but by all the other admissible solutions $U_{m+1}, U_{m+2}, \cdots$ of the Euler equation (0.1).

The chief interest naturally centered in the Jacobi condition, which excludes the possibility of the point conjugate to $x=0$ in the extended sense lying within the interval 0,1 . This condition picks out from the infinite variety of functions $U_{i}$ automatically satisfying the Euler, Legendre and Weierstrass conditions, that particular one, $U_{m}$, which minimizes the integral $D(u)$ under the conditions imposed. This it does by determining the number of oscillations of the function in this interval. In $\$ 2$ of the present memoir important extensions are made in the discussion of the Jacobi condition.

Although in ordinary problems of the calculus both a maximum and a minimum of the function are usually sought, this has not been the case heretofore in problems of the calculus of variations. This is for the good and sufficient reason that one or other of these is infinite; for example, the maximum in the problem (0.3), (0.2), (0.4), (0.5) is infinite; in fact the conditions (0.6), (0.7) are interpreted to mean that no maximum is possible. In contradiction to these considerations for the ordinary case, some of the problems proposed in this paper possess both maximum and minimum solutions. 
Suppose there be added to $(0.3),(0.2),(0.4),(0.5)$ the infinite number of linear conditions

$$
\int_{0}^{1} k U_{j} u d x=0 \quad(j=s+1, \ldots ; s \geqq m) ;
$$

as is shown in $\$ 3$ the minimum is not affected by the addition of these conditions, being furnished by $U_{m}$ as before. But now a maximum of the integral under the same conditions enters and is given by $U_{s}$. By computing the Legendre and Weierstrass conditions for the infinitely extended problems it is found that they have respectively the forms $(0.6),(0.7)$ as before; this fits in well with the preconceived notions of a minimum but since these conditions in the same form appear with the maximum problem as well, their significance has, for the moment at least, disappeared. This is perhaps more immediately evident if $s$ is chosen equal to $m$. The only function orthogonal to $U_{i}$ for $i=1, \cdots, m-1, m+1, \cdots$, and subject to the conditions (0.2), (0.4) is $U_{m}$; this function then furnishes both a maximum and minimum to the integral $D(u)$, while criteria such as the Legendre and Weierstrass should, by all the rules of the game, be different for the two cases. In the treatment of the ordinary problem* the derivation of the Legendre condition is independent of other conditions such as the Jacobi; the same remark may be made concerning the Weierstrass condition as derived by the discoverer. It is noteworthy that the significance of these two criteria as independent conditions has vanished never to return so far as the problems of this paper are concerned. The Weierstrass necessary condition, however, is sometimes deduced on the hypotheses that the Jacobi condition is satisfied in the interval; and in that fcrm, but for the minimum alone, it survives in the problem here discussed. Naturally the Legendre condition, which may be regarded as a less general form of the Weierstrass, must appear in the same rôle. These conditions might well be listed also in some form in any set of sufficient conditions for a minimum of our problem. On the other hand for the maximum there would appear to be no conditions of the usual nature at all possible beyond the Euler equation.

The expectation that the main interest of the new problem would center around the Jacobi condition concerning the conjugate point is fulfilled. For the minimum problem this criterion is placed along side of the Euler as fundamental. The generalized conjugate point must lie outside the interval for a minimum; for the maximum problem proposed it would then follow

* For example, see Bolza, Variationsrechnung. This admirable treatise is a mine of information, and the author wishes to acknowledge his indebtedness to it. 
as a condition that the conjugate point lie within the interval. Lying without the interval is a definite criterion and naturally serves as one of a series of sufficient conditions; lying within the interval is a much more shadowy condition. Probably the number of conjugate points existing in the interval is significant, but such a criterion would seem to indicate not much more than the number of steps the maximum problem is removed from the minimum problem.

It appears then that for problems with an infinite number of auxiliary conditions imposed on the function it is to be expected that a generalization of the Euler conditions will retain its importance for both sorts of extrema, and that the generalization of the Jacobi condition will be vital for scrutinizing the various possibilities that present themselves as solutions of the Euler equation. For one sort of extremum the Jacobi condition will probably serve both among the necessary and among the sufficient conditions, while for the other sort its significance will be negative only. On the other hand it is to be expected that the conditions arising as limiting cases of the Weierstrass and Legendre conditions will, for one sort of extrema, be relegated to positions subsidiary to the Jacobi condition, and for the other be dropped out of consideration.

One might go a step further in indicating the breakdown of necessary conditions in problems with an infinite number of auxiliary conditions. In relative maxima and minima of two quadratic forms a necessary and sufficient condition for the existence of an extremum is that one of these forms be definite; which one does not matter. In the present discussion it is not necessary that $p$ be of one sign in order that the integral $(0.3)$ have an extremum. For example consider the problem

$$
\begin{aligned}
& \int_{0}^{1}(1-2 x) y^{\prime 2} d x=\text { extremum, } y(0)=y(1)=0, \\
& \int_{0}^{1} 2 y^{2} d x=1, \int_{0}^{1} y \sin n \pi x d x=0 \quad(n=3,4, \cdots),
\end{aligned}
$$

where only those functions $y(x)$ are to be considered which are continuous and the square of whose derivative is integrable. It may be noted that the only functions satisfying the auxiliary conditions are $c_{1} \sin \pi x+c_{2} \sin 2 \pi x$, $c_{1}^{2}+c_{2}^{2}=1$. On setting this family of functions in the integral to be made an extremum, there results a quadratic form in the variables $c_{1}, c_{2}$ from which with the relation $c_{1}{ }^{2}+c_{2}{ }^{2}=1$ the problem may be solved. It would appear that in this case none of the usual necessary conditions have any significance, 
not even the Euler condition. In this respect the problem bears some resemblance to the special case of the ordinary isoperimetric problem where the formal solution is a minimizing extremal for the integral involved in the auxiliary condition.

Courant has shown* that if in the problem $(0.3),(0.2),(0.4),(0.5)$ the linear conditions $(0.5)$ be replaced by others more general

$$
\int_{0}^{1} V_{i} u d x=0 \quad(i=1,2, \cdots, m-1),
$$

where $V_{i}(x)$ are arbitrary continuous functions, and if the minimum (or lower bound) of $D(u)$ be denoted by $D\left(V_{1}, \cdots, V_{m-1}\right)$ this minimum cannot be greater than that of the original problem. In other words $\lambda_{m}$ is a minimax, that is the maximum of $D\left(V_{1}, \cdots, V_{m-1}\right)$ which is itself the minimum of $D(u)$ under the conditions $(0.2),(0.4),(0.9)$. Obviously $U_{1}$ furnishes a minimin $\lambda_{1}$, that is a minimum of $D\left(V_{1}, \cdots, V_{m-1}\right)$. If there are a denumerable infinity of the conditions (0.9), there can be no minimax, but the minimin is still $\lambda_{1}$.

If the conditions (0.9) are divided into two groups $1, \cdots, l-1$; $l, \cdots, m-1$, the minimum of $D(u)$ will still be a function $D\left(V_{1}, \cdots, V_{m-1}\right)$; this may be maximized for $V_{1}, \cdots, V_{l-1}$, and minimized for $V_{l}, \cdots, V_{m-1}$, the function $U_{l}$ giving a minimaximin $\lambda_{l}$.

In the case of both minimum and maximum of $D(u)$ under the conditions (0.2), (0.4), (0.5), (0.8) the Euler equation obtained in a formal manner is

$$
\left(p u^{\prime}\right)^{\prime}+q u+\lambda k u-\sum_{1}^{m-1} \mu_{i} k U_{i}-\sum_{i+1}^{\infty} \mu_{i} k U_{i}=0
$$

with solutions

$$
\alpha u_{1}(x, \lambda)-\sum_{1}^{m-1} \frac{\mu_{i}}{\lambda-\lambda_{i}} U_{i}-\sum_{i+1}^{\infty} \frac{\mu_{i}}{\lambda-\lambda_{i}} U_{i},
$$

representing an infinity-parameter family of extrema vanishing at $x=0$, the function $u_{1}(x, \lambda)$ being a solution of the homogeneous equation ( 0.1$)$ which vanishes at $x=0$. It is shown later that $\mu_{i}=0$ for all the minimizing and maximizing extremals; in other words these extremals are solutions of the homogeneous equation (0.1).

In discussing the necessity of the Euler equations it may be noted that in the infinite problem the variations which are admitted by the conditions

- R. Courant and D. Hilbert, Methoden der Mathematischen Physik, p. 325. 
(0.5) must be linearly dependent on $U_{m}, U_{m+1}, \cdots$, so that any function which rannot be expanded in terms of this partial set of orthogonal functions is barred from consideration. In the problem of this paper admissible variations must be linearly dependent on $U_{m}, \cdots, U_{*}$. The family may thus be written

$$
\eta=\beta u_{1}(x, \lambda)+\sum_{m}^{\dot{n}} \beta_{i} U_{i}
$$

It is significant that the only function common to this family of admissible variations and the family of extremals $(0.10)$ is the minimizing extremal $u_{1}(x, \lambda)$.

In many respects the problems of this paper resemble those of relative extrema in quadratic forms involving a finite number or infinite number of variables. The imposition of auxiliary conditions may be regarded as reducing the number of degrees of freedom; when an infinite number of degrees of freedom are taken away there may be a finite or an infinite number remaining. To pursue this notion further let us consider $\sin n \pi x$ as a basic set of functions in terms of which an arbitrary function $u(x)$ vanishing at $x=0$ and $x=1$ is to be expanded in the interval, and set up the corresponding problem of relative extrema in quadratic forms in an infinity of variables. Set

$$
\begin{aligned}
u(x)=\sum_{1}^{\infty} c_{i} \sin i \pi x, \quad U_{l}(x) & =\sum_{1}^{\infty} d_{i}^{(l)} \sin i \pi x \\
(l & =1,2, \cdots, m-1 ; s+1, \cdots) .
\end{aligned}
$$

The problem is to determine the $c$ 's so that the quadratic form

$$
\begin{array}{r}
\int_{0}^{1}\left[p \sum_{i} i c_{i} \cos i \pi x \sum_{i} j c_{i} \cos j \pi x-q \sum_{i} c_{i} \sin i \pi x \sum_{i} c_{j} \sin j \pi x\right] d x \\
\equiv \sum_{i j} e_{i j} c_{i} c_{j}
\end{array}
$$

is an extremum under the quadratic condition

$$
\int_{0}^{1}\left[k \sum_{i} c_{i} \sin i \pi x \sum_{i} c_{i} \sin j \pi x\right] d x \equiv \sum_{i j} g_{i j} c_{i} c_{i}=1,
$$

and the infinite number of linear conditions

$$
\int_{0}^{1}\left[k \sum_{i} d_{i}^{(l)} \sin i \pi x \sum_{j} c_{j} \sin j \pi x\right] d x \equiv \sum_{i j} h_{i j} c_{i} d_{i}^{(l)}=0 .
$$

This leads formally to the problem of finding an extremum for 


$$
\sum_{i j} e_{i j} c_{i} c_{i}+\lambda \sum_{i j} g_{i j} c_{i} c_{j}+\sum_{l} \mu_{l} \sum_{i j} h_{i j} c_{i} d_{i}^{(l)}
$$

subject to the conditions (0.12), (0.13) and on differentiation with regard to the $c$ 's and the $\mu$ 's gives rise to the linear equations

$$
\sum_{i}\left(e_{i j}+\lambda g_{i j}\right) c_{i}+\sum_{l} \mu_{l} \sum_{i} h_{i j} d_{i}^{(l)}=0 \quad(i=1,2, \cdots)
$$

together with (0.13). In order that this infinity of linear homogeneous equations in $c$ 's and $\mu$ 's have a solution it is necessary that $\lambda$ be a root of an infinite determinant consisting of four groups, each of infinite extent in both directions. This may be written

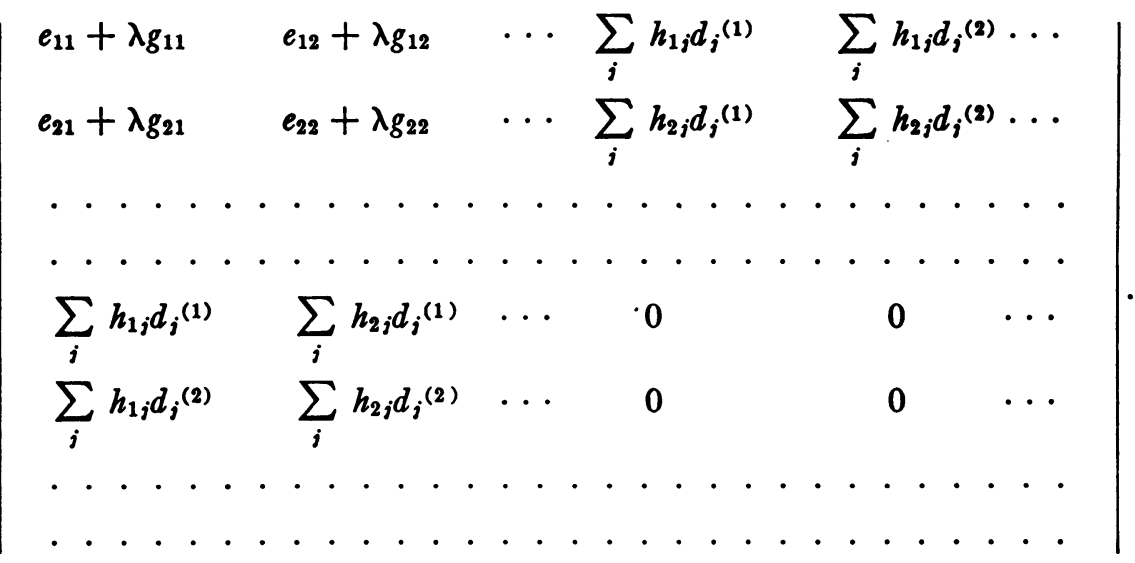

The quadratic condition (0.12) fixes the multiplicative constant involved in the solution of the homogeneous equations. Since from the method of definition, $e_{i j}=e_{j i}, g_{i j}=g_{j i}, h_{i j}=h_{j i}$ the determinant is symmetric. Since it is known in advance (\$3) that both the maximum and minimum problems have solutions, the infinite-bordered determinant must have $s-m+1^{*}$ roots $\lambda_{i}$. For these values the solutions of the linear equations $(0.12),(0.14)$ furnish the various sets of $c$ 's which give not only the solutions $U_{0}, U_{m}$ of the problems but also the other functions $U_{m+1}, \cdots, U_{s-1}$.

In studying these problems of maximizing and minimizing the quadratic form (0.11) under the quadratic condition (0.12) and the infinite number of linear conditions $(0.13)$, the question naturally presents itself as to

* For the minimum problem in the polar case the interesting situation develops that the determinant corresponding to (0.15) has an infinite number of roots $\lambda$, each of which is known in advance, and for each of which the equations (0.14) have solutions. 
what is the condition (analogous in some respects to the Jacobi criterion in the calculus of variations) which picks out, in one case, $U_{\text {, }}$, and in the other, $U_{m}$, from the various possibilities $U_{m}, \cdots, U_{\text {. }}$. For the same problem in a finite number of variables the author has derived this condition*; that discussion suggests an analogous theorem here.

If instead of $\sin n \pi x$ the functions $U_{i}$ are used as basic system the treatment is much simplified. As may be seen from the discussion in $\$ 3$ all terms of the determinant (0.15) vanish except those in the main diagonal of each of the three non-zero divisions.

To indicate the connection $\uparrow$ with the theory of integral equations, denote by $G(x, \xi)$ the Green's function of the differential expression

$$
M(u) \equiv\left(p u^{\prime}\right)^{\prime}+q u,
$$

corresponding to the boundary conditions $(0.2)$. Then the integral equation

$$
u(x)=\lambda \int_{0}^{1} k(\xi) G(x, \xi) u(\xi) d \xi
$$

has the same solutions as the system (0.1), (0.2).

On setting $M(u)=h(x)$, we have from the known properties of the Green's function

$$
u(x)=-\int_{0}^{1} G(x, \xi) h(\xi) d \xi .
$$

On the other hand, integration by parts gives

$$
D(u)=-\int_{0}^{1} u M(u) d x=\int_{0}^{1} \int_{0}^{1} G(x, \xi) h(x) h(\xi) d x d \xi .
$$

Thus the discussion of the extrema of the integral $D(u)$ is reduced to that of the integral on the right of (0.19). If we multiply (0.17) by $k(x) u(x)$ and integrate, we obtain the formula

$$
K_{0}(x) \equiv \int_{0}^{1} k u^{2} d x=\lambda \int_{0}^{1} \int_{0}^{1} k(x) k(\xi) G(x, \xi) u(x) u(\xi) d x d \xi \equiv \lambda R(u),
$$

and hence from (1.10) we have, when $U_{i}$ is a characteristic function,

- Relative extrema of pairs of quadratic and hermitian forms, these Transactions, vol. 26, p. 491.

$\dagger$ I am indebted to my colleague, Professor J. Tamarkin, for suggestions concerning the methods used in connection with these expansions. 


$$
D\left(U_{i}\right)=\lambda_{i} K_{0}\left(U_{i}\right)=\lambda_{i}{ }^{2} R\left(U_{i}\right) .
$$

When $q \leqq 0$ the integral $D$ is positive and hence the integral $R$ is also positive.

In $\$ 3$ the three integrals $D(u), K_{0}(u)$, and $R(u)$ are discussed in regard to relative maxima and minima under an infinite number of linear auxiliary conditions.

The Jacobi condition as discussed in $\$ 6$ concerns the non-vanishing of an infinite determinant involving integrals. When any finite number of conditions are dropped from the set of linear conditions (0.8), the infinite determinant corresponding to the resulting problem has again no zero in the interval 0,1 , and it is a curious fact that its ratio to the original is a decreasing function throughout the interval.

A portion of the discussion in this paper is too formal, omitting much in the way of justification of infinite processes. Since, however, the extrema actually exist, the main argument is correct and the briefer treatment has its advantages.

It may be noted further that the linear character of all except one of the auxiliary conditions renders the treatment much simpler than it would be in the general case. In particular the analogons of the Legendre and Weierstrass criteria and of the Hamilton function and Hilbert integral have very simple forms.

The results of this paper as here given for the simple boundary conditions (0.2) may be extended without difficulty to more complicated cases. The treatment as given for one independent variable may be readily generalized to regions of two or more dimensions. With the exception of the process of taking the derivatives of the quotients of the determinants arising in the discussion of the Jacobi condition, all notions and methods go over almost without change to the more general problem. The interpretation of the Jacobi condition in terms of oscillation theorems for two or more independent variables, however, is obscure and difficult and has not been worked out.

\section{Preliminary theorems aND Formulas}

In this section we shall assemble some fundamental formulas for later reference and shall review some of the considerations of the paper* which treats the case of a finite number of auxiliary conditions.

Basic for the argument is the self-adjoint differential equation of the second order

$$
L(u) \equiv\left(p(x) u^{\prime}(x)\right)^{\prime}+q(x) u(x)+\lambda k(x) u(x)=0,
$$

\footnotetext{
* Loc. cit., Mathematische Annalen, vol. 68, p. 269
} 
with the boundary conditions

$$
u(0)=u(1)=0,
$$

where $p>0$, and where $p, q$ and $k$ are analytic functions* of $x$ in the interval 0,1 considered.

The general solution $\alpha u_{1}(x, \lambda)+\beta u_{2}(x, \lambda)$ of (1.1) contains two arbitrary constants besides the parameter $\lambda$. Since the discussion of this paper concerns only the family through $x=0, u_{1}$ may be chosen so as to vanish there and the solution may then be written

$$
u=\alpha u_{1}(x, \lambda)
$$

where it is assumed for the sake of uniformity and without loss of generality that $\alpha>0, u^{\prime}(0, \lambda)>0$. As $|\lambda|$ increases all the zeros of $u_{1}(x, \lambda)$ (except that at $x=0)$ move to the left.

As noted in the Introduction, there are two important cases connected with the problems of the calculus of variations. In the orthogonal case there is an infinite set

$$
U_{1}, U_{2}, \cdots
$$

of solutions of (1.1) (1.2) and in the polar case there are two such sets

$$
U_{1}, U_{2}, \cdots ; \quad U_{-1}, U_{-2}, \cdots .
$$

Solutions can be considered orthogonalized and normalized:

(1.6) $\int_{0}^{1} k U_{i} U_{j} d x=0(i \neq j) ; \int_{0}^{1} k U_{i}^{2} d x=1 \quad\left[\int_{0}^{1} k U_{-i}^{2} d x=-1\right]$.

For the orthogonal case, the equation (1.1) is the Euler condition for the calculus of variations problem

$$
D(u) \equiv \int_{0}^{1}\left(p u^{\prime 2}-q u^{2}\right) d x=\min ., \quad u(0)=u(1)=0,
$$

subject to the quadratic auxiliary condition

$$
K_{0} \equiv \int_{0}^{1} k u^{2} d x=+1 .
$$
tions.

- The main features of the discussion can be carried through under much less stringent condi- 
For, on setting

$$
v_{0}(x)=\int_{0}^{x} k u^{2} d x, \text { and hence } v_{0}^{\prime}-k u^{2}=0, v_{0}(0)=0, v_{0}(1)=1,
$$

an application of the Lagrange method transforms the relative minimum problem into that of finding an absolute minimum of the integral

$$
\int_{0}^{1}\left[p u^{\prime 2}-q u^{2}+\lambda\left(v_{0}^{\prime}-k u^{2}\right)\right] d x
$$

having for Euler condition the equation (1.1).

The solution of the minimum problem must then be found amon $g(1.4)$; from the formulas easily derived from (1.1),

$$
\int_{0}^{1}\left(p u^{\prime 2}-q u^{2}\right) d x=\lambda \int_{0}^{1} k u^{2} d x
$$

it follows that the minimum value is one of the $\lambda$ 's. Since all the other conditions of the minimum problem are satisfied by any of the functions $U_{1}, U_{2}, \cdots$, it must be the Jacobi criterion alone which determines that particular one, $U_{1}$, having no zero within the interval.

If the extremum problem (1.7), (1.8) is changed by the addition of the linear conditions

$$
K_{i} \equiv \int_{0}^{1} k U_{i} u d x=0 \quad(i=1,2, \cdots, m-1),
$$

the solution $U_{1}$ is barred from consideration. On setting

$$
v_{i}=\int_{0}^{x} k U_{i} u d x, \text { and hence } v_{i}^{\prime}-k U_{i} u=0, v_{i}(0)=v_{i}(1)=0,
$$

and considering the problem of minimizing the integral

$$
\int_{0}^{1}\left[p u^{\prime 2}-q u^{2}+\lambda\left(v_{0}^{\prime}-k u^{2}\right)+\sum_{1}^{m-1} 2 \mu_{i}\left(v_{i}^{\prime}-k U_{i} u\right)\right] d x,
$$

the Euler equation takes the non-homogeneous form

$$
\left(p u^{\prime}\right)^{\prime}+q u+\lambda k u+\sum_{1}^{m-1} \mu_{i} k U_{i}=0
$$

with solutions

$$
u=\alpha u_{1}(x, \lambda)-\sum_{1}^{m-1} \frac{\mu_{i} U_{i}}{\lambda-\lambda_{i}}
$$


as may be proved by substitution. This family of curves may be used as the extremals of the problem. It is possible to show that for the minimizing extremal of this family all the $\mu$ 's are zero. For, on setting the value of $u$ from (1.14) in (1.11), using the boundary conditions and the relations (1.6), we find that

$$
0=v_{i}(1)=-\frac{\mu_{i}}{\lambda-\lambda_{i}} \int_{0}^{1} k U_{i}^{2} d x=-\frac{\mu_{i}}{\lambda-\lambda_{i}} \quad(i=1, \cdots, m-1),
$$

from which it follows that $\mu_{i}=0$. The differential equation of the minimizing extremals is thus reduced from (1.13) to (1.1). The Jacobi condition selects the solution which is in this case $U_{m}$ with $m-1$ zeros within the interval.

It should be noted that for some purposes, such as the Jacobi condition, it is well to interpret the family of extremals as being in higher dimensional space. By adding to the two dimensions $x u$ of (1.14), a third $v_{0}$ given by (1.9) and $m-1$ more $v_{i}$ given by (1.12), the extremals may be considered to be curves in the $(m+2)$-dimensional $x u v_{0} v_{i}$ space.

For the polar case, there are two sets of calculus of variations problems for which the equation (1.1) is the Euler condition. One is precisely that of the formulas (1.7) to (1.15); the other is set up by replacing the quadratic condition (1.8) by $K_{0}=-1$.

Let $f(x)$ be any function which vanishes at $x=0$ and $x=1$ and which can be represented in the form

$$
f(x)=\int_{0}^{x} \phi(x) d x
$$

where $\phi(x)$ is integrable together with its square. It is known* that $f(x)$ can be expanded in an absolutely and uniformly convergent series (in both the orthogonal and the polar case):

$$
f(x)=\sum f_{i} U_{i}(x), f_{i}=\operatorname{sign} \lambda_{i} \int_{0}^{1} k f U_{i} d x,
$$

the summation being taken over all the characteristic values. Substituting here $f(\xi)=G(x, \xi)$ and observing from (0.17) that

$$
U_{i}(x)=\lambda_{i} \int_{0}^{1} k(\xi) G(x, \xi) U_{i}(\xi) d \xi,
$$

* J. Tamarkine, Problème du developpement d'une fonction arbitraire en séries de Sturm-Liouville, Comptes Rendus, vol. 156 (1913), pp. 1589-1591; L. Lichtenstein, Zur Analysis der unendlichvielen Variabeln, Rendiconti del Circolo Matematico di Palermo, vol. 38 (1914), pp. 113-166. 
it is readily seen that

$$
G(x, \xi)=\sum \frac{U_{i}(x) U_{i}(\xi)}{\left|\lambda_{i}\right|}
$$

the series being absolutely and uniformly convergent.

If $h(x)$ be an integrable function, on multiplying (1.18) by $h(x) h(\xi)$ and integrating we get the bilinear formula

$$
\int_{0}^{1} \int_{0}^{1} G(x, \xi) h(x) h(\xi) d x d \xi=\sum \frac{h_{i}^{2}}{\left|\lambda_{i}\right|} ; \quad h_{i}=\int_{0}^{1} h U_{i} d x .
$$

Let us now identify $h(x)$ with $M(u)$ as defined in (0.16). If $u(x)$ is a function whose first derivative is absolutely continuous in 0,1 , then $u^{\prime \prime}(x)$ exists almost everywhere and is integrable. Hence from (0.19) and (1.19) we have $D(u)=\sum\left(h_{i}{ }^{2} /\left|\lambda_{i}\right|\right)$, and since from (1.19), (1.17), and (0.18)

$$
h_{i}=\lambda_{i} \int_{0}^{1} \int_{0}^{1} h(x) k(\xi) G(x, \xi) U_{i}(\xi) d x d \xi=-\lambda_{i} \int_{0}^{1} k(\xi) u(\xi) U_{i}(\xi) d \xi,
$$

this may be written

$$
D(u)=\sum\left|\lambda_{i}\right| c_{i}^{2}, \quad c_{i}=\operatorname{sign} \lambda_{i} \int_{0}^{1} k(x) u(x) U_{i}(x) d x .
$$

We note also that from (1.16) there follows

$$
K_{0}(f) \equiv \int_{0}^{1} k f^{2} d x=\sum \operatorname{sign} \lambda_{i} f_{i}^{2} ; K_{0}(u)=\sum \operatorname{sign} \lambda_{i} c_{i}^{2} .
$$

Some relations between the various solutions of (1.1) are important and will now be developed. It may be noted that the function $u(x, \lambda)$ satisfies the equation

$$
\left(p \frac{\partial u^{\prime}}{\partial \lambda}\right)+(q+\lambda k) \frac{\partial u}{\partial \lambda}+k u=0, \quad \frac{\partial u(0)}{\partial \lambda}=0 .
$$

On multiplication of (1.1) for the characteristic number $\lambda_{m}$ and solution $U_{m}$ by $\partial u / \partial \lambda$ and of (1.22) by $-U_{m}$, addition and integration, we obtain the relation

$$
U_{m}^{\prime} \frac{\partial u}{\partial \lambda}-U_{m} \frac{\partial u^{\prime}}{\partial \lambda}=\frac{\lambda-\lambda_{m}}{p} \int_{0}^{x} k U_{m} \frac{\partial u}{\partial \lambda} d x+\frac{1}{p} \int_{0}^{x} k U_{m} u d x .
$$

which for the special case $u=U_{1}$ and $m=1$ becomes

$$
p\left(u^{\prime} \frac{\partial u}{\partial \lambda}-u \frac{\partial u^{\prime}}{\partial \lambda}\right)=\int_{0}^{x} k u^{2} d x .
$$


If $U_{m}, U_{l}$ denote any two of the family (1.4) (or (1.5)) corresponding to the parameters $\lambda_{m}, \lambda_{l}$ it may be proved in a similar manner that they satisfy the identity

$$
U_{m}^{\prime} U_{l}-U_{m} U_{l}^{\prime}=\frac{\lambda_{l}-\lambda_{m}}{p} \int_{0}^{x} k U_{m} U_{l} d x .
$$

If $\eta$ is a continuous function vanishing at 0 and $x_{1}$, on multiplication of $L(u)=0$ by $\eta$ and integration by parts there results a relation $D(u, \eta)$ $=\lambda K_{0}(u, \eta)$ between the polar forms $D(u, \eta) \equiv \int_{0}^{z^{\prime}}\left(p u^{\prime} \eta^{\prime}-q u \eta\right) d x, K_{0}(u, \eta)$ $\equiv \int_{0}^{s_{1}} k u \eta d x$. Further, let $\eta$ be an allowable variation in the interval $0, x_{1}$ for the problem (1.7), (1.8), (1.11); that is, let

$$
K_{0}(u, \eta)=0, K_{i} \equiv \int_{0}^{x_{1}} k U_{i} d x=0 ;
$$

then $D(u, \eta)=0$.

\section{An extension of the finite problem. The Jacobi CONDITION AND ITS INTERPRETATION}

Using the method of the earlier paper* let us pursue considerably further than was there necessary the question of the Jacobi condition for the finite case. Consider the new problem of a minimum of $D(u)$ under the boundary conditions $u(0)=u(1)=0$, the quadratic condition $K_{0}=1(1.8)$ and the two sets of linear conditions

(2.1) $K_{i} \equiv \int_{0}^{1} k U_{i} u d x=0, i=1, \cdots, m-1 ; i=s+1, \cdots, l ; s \geqq m$.

The addition of the second set of (2.1) cannot decrease the minimum; that it is not increased is readily seen by noting that the function furnishing the minimum for the first set (2.1) only is $U_{m}$ and that this function also satisfies the second set. That the minimum is $\lambda_{m}$ furnished by $U_{m}$ may also be proved in a manner analogous to Theorem IV of $\$ 3$. In other words the second set of conditions (2.1) affects the problem only formally. The Euler equation takes the form

$$
\left(p u^{\prime}\right)^{\prime}+q u+\lambda k u+\sum_{1}^{m-1} \mu_{i} k U_{i}+\sum_{i+1}^{l} \mu_{i} k U_{i}=0,
$$

with the $(m+l-s+1)$-parameter family of solutions through the origin

* Loc. cit., Mathematische Annalen, vol. 68, p. 289. 


$$
u=\alpha u_{1}(x, \lambda)-\sum_{1}^{m-1} \frac{\mu_{i} U_{i}}{\lambda-\lambda_{i}}-\sum_{i+1}^{l} \frac{\mu_{i} U_{i}}{\lambda-\lambda_{i}},
$$

where $\alpha u_{1}(x, \lambda)$ is defined as in (1.3). By a method similar to that used in $\$ 1$, the plane family of extremals $(2.3)$ may be replaced by an $(m+l-s+1)$ parameter $\left(\alpha \lambda \mu_{i}\right)$-family in $(m+l-s+2)$-dimensional $\left(x u v_{0} v_{i}\right)$-space

$$
\begin{aligned}
& u=\alpha u_{1}(x, \lambda)-\sum \mu_{i} \frac{U_{i}}{\lambda-\lambda_{i}}, v_{0}=\int_{0}^{x} k\left(\alpha u_{1}-\sum \frac{\mu_{i} U_{i}}{\lambda-\lambda_{i}}\right)^{2} d x, \\
& v_{j}=\int_{0}^{x} k\left(\alpha u_{i}-\sum \frac{\mu_{i} U_{i}}{\lambda-\lambda_{i}}\right) U_{j} d x(j=1, \cdots, m-1 ; s+1, \cdots, \eta),
\end{aligned}
$$

and passing through the origin $(0,0, \cdots, 0)$. The summation over $i$, here as hereafter, is supposed to extend through the range $1, \cdots, m-1$, $s+1, \cdots, l$. It may be shown as in $\$ 1$ that in this family (2.4) there is imbedded the space curve corresponding to the minimizing extremal $u=U_{m}\left(x, \lambda_{m}\right)$ and for which $\mu_{i}=0, \lambda=\lambda_{m}$. Geometrically interpreted, the Jacobi condition demands that within the $x$-interval 0,1 this space curve be not cut by any of its neighbors. This is equivalent to saying that the $m+l-s+1$ homogeneous equations in as many unknowns

$$
\begin{gathered}
\delta \lambda\left[\alpha \frac{\partial u_{1}\left(x, \lambda_{m}\right)}{\partial \lambda}\right]+\delta \alpha\left[u_{1}\left(x, \lambda_{m}\right)\right]-\sum \delta \mu_{i}\left(\frac{U_{i}(x)}{\lambda_{m}-\lambda_{i}}\right)=0, \\
(2.5) 2 \alpha^{2} \delta \lambda \int_{0}^{x} k u_{1} \frac{\partial u_{1}}{\partial \lambda} d x+2 \alpha \delta \alpha \int_{0}^{x} k u_{1}^{2} d x-\sum \frac{2 \alpha \delta \mu_{i}}{\lambda_{m}-\lambda_{i}} \int_{0}^{x} k u_{1} U_{i} d x=0, \\
\alpha \delta \lambda \int_{0}^{x} k U_{j} \frac{\partial u_{1}}{\partial \lambda} d x+\delta \alpha \int_{0}^{x} k u_{1} U_{j} d x-\sum\left(\frac{\delta \mu_{i}}{\lambda_{m}-\lambda_{i}}\right) \int_{0}^{x} k U_{i} U_{j} d x=0
\end{gathered}
$$

have no solution for $0<x<1$. The value of $x$ next after $x=0$ for which these equations hold is called the conjugate point in the extended sense. And the Jacobi condition demands that this conjugate point lie beyond the point $x=1$. Now an infinite set $U_{m}, \cdots, U_{s}, U_{l+1}, \cdots$ of characteristic solutions of (1.1) satisfy all the others of the set of sufficient conditions; hence it is the Jacobi condition alone which selects $U_{m}$ as the minimum. It will later be shown that the condition implies that $u_{1}$ vanish $m-1$ times in the interval, thus identifying it with $U_{m}$ except for a constant multiplier.

On the analytic side, the Jacobi condition concerns the sign of the second variation. For the purpose of calculating the second variation, we may take the integral in the form 


$$
\int_{0}^{1}\left[p u^{\prime 2}-q u^{2}+\lambda_{m}\left(v_{0}^{\prime}-k u^{2}\right)+\sum 2 \mu_{i}\left(v_{i}^{\prime}-k U_{i} u\right)\right] d x .
$$

The admissible variation $\eta$ is subject to the restrictions $\eta(0)=\eta(1)=0$ and

$$
\int_{0}^{1} k u \eta d x=0, \quad \int_{0}^{1} k U_{\eta \eta} d x=0(i=1, \cdots, m-1 ; s+1, \cdots, l) .
$$

After the usual computation we find

$$
\delta^{2} D=\epsilon^{2} \int_{0}^{1}\left(p \eta^{\prime 2}-q \eta^{2}-\lambda_{m} k \eta^{2}\right) d x
$$

which by integration by parts and addition of multiples of the linear terms (2.6) becomes

$$
\delta^{2} D=\epsilon^{2} \int_{0}^{1}-\eta\left[\left(p \eta^{\prime}\right)^{\prime}+q \eta+\lambda_{m} k \eta+\alpha \delta \lambda k u+\sum \mu_{i} k U_{i}\right] d x .
$$

Consider the expression inside the brackets of this integrand; it will vanish if for $\eta$ we substitute the left hand side of the first line of (2.5), as can be proved by substitution and use of (1.1) and (1.22) and remembering that $\mu_{i}=\delta \mu_{i}$. It follows that if $x=1$ is the point conjugate to $x=0$, the second variation $\delta^{2} D$ may be made zero by giving to $\eta$ this value.

A similar argument may be applied to any interval $0, x_{1}$, where $x_{1}$ is the point conjugate to 0 . If $x_{1}$ is within the interval 0,1 , and if $\eta$ is an admissible variation over $0, x_{1}$, we may set $\eta=0$ in the interval $x_{1}, 1$. In that case the conditions (2.6) still hold and the second variation may still be. written in the form (2.8) and may be made to vanish by the same device.

The original minimum problem for $u$ can be put into essentially the same form as (2.7) (2.6) with a proper quadratic restriction. Hence the minimum for $D(\eta) / K_{0}(\eta)$ is $\lambda_{m}$ furnished by $\eta=U_{m}(x)$ which is an analytic function. Any variation which is zero in a part of the interval cannot be analytic and hence cannot furnish the minimum for (2.7). In that case $\delta^{2} D$ can be made negative, which indicates that the point conjugate to $x=0$ cannot lie within the interval.

For the sake of definiteness, let us choose $m=2, s=3, l=4$ and proceed to set up in detail the Jacobi condition. The Jacobi determinant of (2.5), apart from a constant factor, is 


$$
D_{14}(x, \lambda)=\left|\begin{array}{cccc}
\frac{\partial u_{1}}{\partial \lambda} & u_{1} & U_{1} & U_{4} \\
\int_{0}^{x} k u_{1}\left(\frac{\partial u_{1}}{\partial \lambda}\right) d x & \int_{0}^{x} k u_{1}^{2} d x & \int_{0}^{x} k u_{1} U_{1} d x & \int_{0}^{x} k u_{1} U_{4} d x \\
\int_{0}^{x} k U_{1}\left(\frac{\partial u_{1}}{\partial \lambda}\right) d x & \int_{0}^{x} k U_{1} u_{1} d x & \int_{0}^{x} k U_{1}^{2} d x & \int_{0}^{x} k U_{1} U_{1} d x \\
\int_{0}^{x} k U_{4}\left(\frac{\partial u_{1}}{\partial \lambda}\right) d x & \int_{0}^{x} k U_{4} u_{1} d x & \int_{0}^{x} k U_{4} U_{1} d x \int_{0}^{x} x U_{1}^{2} d x
\end{array}\right|
$$

and for a minimum the Jacobi condition asserts that this can have no zero within the interval. It vanishes at $x=0$, but not at $x=1$ since at that point its value is $\partial u_{1} / \partial \lambda$ and from (1.24) and (1.6) it is evident that not both $u$ and $\partial u / \partial \lambda$ can vanish at $x=1$.

Add to the conditions of this special problem the further one

$$
\int_{0}^{1} k U_{3} u d x=0 .
$$

The solution is still $U_{2}$, but in place of $D_{14}(x, \lambda)$ there is a five-rowed determinant $D_{134}(x, \lambda)$, which is obtained by inserting between the third and fourth rows of (2.5) a new row similar to these except that $U_{3}$ replaces $U_{1}$ or $U_{4}$ and between the third and fourth column a new column in similar fashion. Schematically this new determinant, which must not vanish within the interval, may be expressed as follows:

$$
D_{184}(x, \lambda)=\left|\begin{array}{lllll}
\frac{\partial u_{1}}{\partial \lambda} & u_{1} & U_{1} & U_{3} & U_{4} \\
a_{2} & a_{22} & a_{23} & a_{24} & a_{25} \\
a_{3} & a_{32} & a_{33} & a_{34} & a_{35} \\
a_{4} & a_{42} & a_{48} & a_{44} & a_{45} \\
a_{5} & a_{52} & a_{53} & a_{54} & a_{55}
\end{array}\right| .
$$

Before proceeding further with the main argument, let us prove a fundamental lemma, the compact form of the proof of which is due to my colleague, Professor H. P. Manning.

LEMma. Given two determinants, $D_{m}$ and $D_{m+1}$, of the mth and $(m+1)$ th orders, respectively, the first being a first minor of the second,

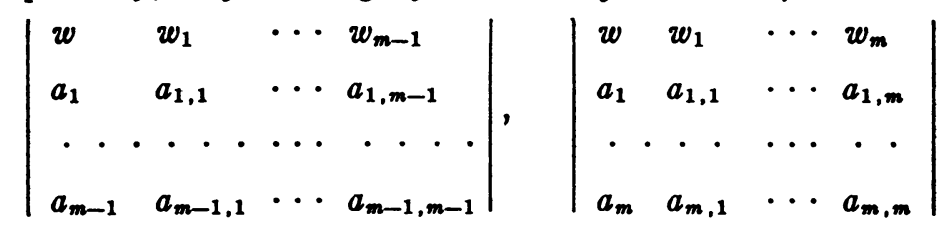


and the terms being subject to the following conditions:

$$
a_{i j}=a_{j i}, w w_{i}^{\prime}-w^{\prime} w_{i}=a_{1 i} f(x)+l_{i} a_{i}, w_{i} w_{j}^{\prime}-w_{j}^{\prime} w_{i}=\left(l_{i}-l_{i}\right) a_{i j},
$$

and such that the derivatives of the terms of any row other than the first form multiples of the first. Denoting by $\alpha, \alpha_{1}, \cdots, \alpha_{m-1}$ the cofactors of the first row of $D_{m-1}$ and by $A, A_{1}, \cdots, A_{m}$ those of the first row of $D_{m}$ and for completeness of notations setting $\alpha_{m}=0$, then

$$
\frac{d}{d x} \frac{D_{m}}{D_{m-1}}=\frac{l_{m} A_{m}^{2}}{D_{m-1}^{2}} .
$$

From the theory of determinants the following identities may be written down:

$$
\begin{array}{rlr}
\text { (2.11) } a_{j} \alpha+\sum_{i=1}^{m} \alpha_{i} a_{j i}=0(j=1, \cdots, m-1) ; a_{m} \alpha+\sum_{i=1}^{m} \alpha_{i} a_{m i}=A_{m} ; \\
\text { (2.12) } a_{j} A+\sum_{i=1}^{m} A_{i} a_{j i}=0 & \quad(j=1, \cdots, m) .
\end{array}
$$

Since by hypothesis the determinants formed by replacing any row except the first by its derivative are zero, the numerator of the derivative of the quotient of the determinants can be written in the form of a single determinant of the second order

$$
\left|\begin{array}{cc}
w \alpha+w_{1} \alpha_{1}+\cdots+w_{m} \alpha_{m} & w^{\prime} \alpha+w_{1}^{\prime} \alpha_{1}+\cdots+w_{m}^{\prime} \alpha_{m} \\
w A+w_{1} A_{1}+\cdots+w_{m} A_{m} & w^{\prime} A+w_{1}^{\prime} A_{1}+\cdots+w_{m}^{\prime} A_{m}
\end{array}\right|
$$

In expanding (2.13) the terms involving either $w$ or $w^{\prime}$ may be written $\sum\left(w w_{i}^{\prime}-w^{\prime} w_{i}\right)\left(\alpha A_{i}-A \alpha_{i}\right)$. By hypothesis this becomes

$$
\begin{aligned}
& f(x) \sum a_{1 i}\left(\alpha A_{i}-A \alpha_{i}\right)+\sum a_{i} l_{i}\left(\alpha A_{i}-A \alpha_{i}\right) \\
& \quad \equiv f(x)\left[\alpha\left(a_{1} A+\sum a_{1 i} A_{i}\right)-A\left(a_{1} \alpha+\sum a_{1 i} \alpha_{i}\right)\right]+\sum l_{i} a_{i}\left(\alpha A_{i}-A \alpha_{i}\right)
\end{aligned}
$$

which by (2.11) and (2.12) becomes

$$
\sum a_{i} l_{i}\left(\alpha A_{i}-A \alpha_{i}\right) \text {. }
$$

The other terms of the expansion of (2.13) are $\sum_{i j}\left(w_{i} w_{i}^{\prime}-w_{i}^{\prime} w_{j}\right)\left(\alpha_{i} A_{i}-\alpha_{j} A_{i}\right)$ $(j>i)$ which by hypothesis may be written

$$
\begin{aligned}
\sum_{i j}\left(l_{j}-l_{i}\right) a_{i j}\left(\alpha_{i} A_{j}-\alpha_{j} A_{i}\right) & (j>i) \\
=\sum_{i j}\left[l_{j} \alpha_{i} A_{j}+l_{i} \alpha_{j} A_{i}-l_{j} \alpha_{j} A_{i}-l_{i} \alpha_{i} A_{j}\right] a_{i j} & (j>i) \\
& =\sum_{i} l_{j} A_{i} \sum \alpha_{i} a_{i j}-\sum l_{j} \alpha_{j} \sum_{i} A_{i} a_{i j} \text { (i and } j \text { independent). }
\end{aligned}
$$


Adding this last expression to (2.14) we have for the numerator of the determinant

$$
\sum_{i} l_{j} A_{i}\left(\alpha a_{i}+\sum_{i} \alpha_{i} a_{i j}\right)-\sum_{i} l_{j} \alpha_{i}\left(A \alpha_{i}+\sum_{i} A_{i} a_{i j}\right) .
$$

By (2.11) and (2.12) this reduces to $l_{m} A_{m}{ }^{2}$. The derivative is thus $l_{m} A_{m}{ }^{2} / D_{m-1}^{2}$ and has the sign of $l_{m}$.

Further it may be noted that if from $D_{m}$ we pick out another minor $E_{a t}$ by leaving out any row (the $s$ th) except the first and any column (the $t$ th) except the first, the same argument holds and we find that

$$
\frac{d}{d x} \frac{D_{m}}{E_{s t}}=l_{s} \frac{A_{g} A_{t}}{E_{a t}{ }^{2}}
$$

The argument may also be applied in a formal fashion when $m$ is infinite. Returning now to the main discussion it is possible to write down the derivative with regard to $x$ of the quotient of (2.10) and its first minor (2.9). As may be seen from (1.23), (1.24), (1.25), the conditions of the lemma are satisfied by the determinants (2.9), (2.10).

Hence we have

$$
\frac{d}{d x} \frac{D_{14}}{D_{184}}=\frac{\lambda_{2}-\lambda_{8}}{p D_{184}^{2}} \alpha s^{2}<0,
$$

where $\alpha_{3}$ is the cofactor of $U_{3}$ in (2.10).

Now $D_{134}$ and $D_{14}$ vanish at $x=0$, do not vanish in the interval, and have at $x=1$ the same value

$$
\left.\frac{\partial u_{1}(x, \lambda)}{\partial \lambda}\right|_{x-1, \lambda-\lambda_{2}}
$$

which is positive as may be noted from (1.24), since $U_{2}(1)=0$ and $U_{2}^{\prime}>0$, this being the second zero beyond $x=0$ for this function.

The formula (2.15) indicates that the roots of $D_{134}$ and $D_{14}$ separate each other and since it may be shown as in $\$ 7$ of the paper cited that at $x=0$ the determinant $D_{13}$ has the higher order of zero, the quotient $D_{14} / D_{14}$ starts at $x=0$ with a value $+\infty$ and having a value 1 at $x=1$, vanishes at the first zero of $D_{14}$ which must lie before that of $D_{184}$.

If $D_{1}$ denotes the determinant obtained by omitting the last row and column from (2.9), the same argument shows that

$$
\left.\frac{d}{d x} \frac{D_{1}}{D_{14}}=\frac{\lambda_{2}-\lambda_{4}}{p(x)} \text { (function) }\right)^{2}<0 .
$$


$D_{1}$ vanishes at $x=0$ of lower order than $D_{14}$ and has at $x=1$ the same value; the next root of $D_{1}$ must then lie before that of $D_{14}$.

In descending one step further in the order of the determinant, the argument is somewhat different and coincides with that of the earlier paper. The formal process of finding by means of the Lemma the derivative of the quotient of two determinants is the same but since in all cases the sign of the result depends on $\lambda_{2}-\lambda_{i}$, the derivative is negative when any condition of the second set of (2.1) is involved and positive when all of these are omitted. The ratio of the determinants at $x=0$ is $+\infty$ in the first case and $-\infty$ in the other.

When the last row and column of $D_{1}$ are omitted and the remaining two-rowed determinant denoted by $D$, it was shown in the earlier paper* (and also follows from the discussion here) that

$$
\frac{d}{d x} \frac{D}{D_{1}}=\frac{\lambda_{2}-\lambda_{1}}{p(x)}(\text { function })^{2}>0,
$$

and further that

$$
\left.\frac{d}{d x} \frac{u_{1}}{D}=\frac{1}{p(x)} \text { (function) }\right)^{2}>0,
$$

and from these facts that $u_{1}$ has precisely one zero between $x=0$ and $x=1$. The Jacobi condition is thus vital in the final handling of this calculus of variations problem. Its place among the necessary conditions and among the sufficient conditions is a fundamentally important one.

The determinant $D_{14}$ is obtained from $D_{134}$ by omitting the fourth row and fourth column; if a different four-rowed minor be selected from $D_{134}$ by omitting any row except the first and any column, a formula for the derivative of the quotient of it by $D_{134}$ may be obtained in the same manner. $\dagger$ If the minor selected be symmetrically placed with regard to the main diagonal, the derivative will involve the square of a minor as in (2.8); if it is not symmetrically placed this square is replaced by the product of two different minors. This process may be repeated step by step until one arrives at $\boldsymbol{u}_{1}$. The ratio of $D_{134}$ (or of $u$ ) to any minor of any order symmetrical to the diagonal is a function of $x$ monotone in the interval 0,1 and one may descend from $D_{184}$ to $u_{1}$ by ladders different from that used above; but in each case the argument determines the exact number of zeros of $u_{1}$.

\footnotetext{
* Loc. cit., Mathematische Annalen, vol 6e, p. 269.
}

$\dagger$ Cf. the sequel of the lemma. 
Returning to the other end of the series of determinants, if an extra condition is imposed on $u_{1}$ so as to give a six-rowed determinant $D_{1 \mu l}$, including $D_{14}$ as a first minor, we have

$$
\frac{d}{d x} \frac{D_{134}}{D_{184 l}}=\left(\lambda_{3}-\lambda_{l}\right)(\text { function })^{2}<0 .
$$

For the various functions, the first zeros beyond $x=0$ lie in the following order from left to right: $u_{1}, D, D_{1}, D_{14}, D_{134}, D_{134 l}$.

For the general problem with conditions (2.1) the essential facts may be formulated in a fashion similar to that of the special case selected. If the second set is deleted, the determinant $D_{1} \ldots m$ has no zero within 0,1 while $D_{1} \ldots m_{-2}$ has one, $D_{1} \ldots m_{-3}$ has two and $u_{1}$ has $m-1$. But the addition of any group of one or more (and in any order) of the second set (which is more or less supernumary to the problem) gives a determinant with no zero within the interval. The imposition of another condition moves further to the right the zero of the determinant, and this continues step by step until as many conditions are imposed as is desired. It is striking that determinants of integrals of any desired order and with no zero in the interval 0,1 can be built up in this simple fashion. It is also noteworthy that the ratio of the determinant or of any minor symmetrically placed with regard to the main diagonal to any other minor contained in it and also symmetrically placed is a function of $x$ monotone in the interval, provided only that the latter contains the term $u_{1}$.

It may further be remarked that the above discussions apply not only when there are two groups of linear conditions $K_{i}=0$, each with consecutive subscripts, but also when these conditions are taken at random. The minimum is furnished by $U_{p}$ where $p$ is the smallest integer not included among the $i$ 's; the Jacobi condition admits of interpretation as in the case discussed. It is also immediately evident that a minimum would exist if $i$ ran over some sequence not including all the integers but with infinity as a limit. The argument of this section paves the way for the extension of the theory to the infinite case.

\section{The EXISTENCE OF EXTrema}

In $\$ 1$ a single sequence of functions $U_{1}, U_{2}, \ldots$ was defined in the orthogonal case and a double sequence $U_{1}, U_{2}, \cdots ; \cdots, U_{-2}, U_{-1}$ was defined in the polar case as solutions of the differential equation

$$
L(u) \equiv\left(p u^{\prime}\right)^{\prime}+q u+\lambda k u=0
$$


under the boundary conditions

$$
u(0)=u(1)=0 .
$$

The theorems of the present section concerning these functions fall into two groups according as the orthogonal $(k(x)$ one sign) or the polar case $(k(x)$ both signs) is considered. For the polar case it is possible (by the addition of an infinity of linear conditions imposed on the functions $U_{-i}$ ) to establish results in nature similar to those of Theorem I; but the principles involved are sufficiently illustrated by the less complicated formulation here given. For the sake of simplicity in the polar case a further hypothesis is made that all the characteristic solutions are real; this will be the case, for example, if $q(x) \leqq 0$.

The relative extrema here discussed concern three integrals the relations of each of which to the differential equation (3.1) have been discussed in the Introduction. These are

$$
\begin{gathered}
D(u) \equiv \int_{0}^{1}\left(p u^{\prime 2}-q u^{2}\right) d x, p>0 ; K_{0}(u) \equiv \int_{0}^{1} k u^{2} d x, \\
R(u) \equiv \int_{0}^{1} \int_{0}^{1} k(x) k(\xi) G(x, \xi) u(x) u(\xi) d x d \xi,
\end{gathered}
$$

where $G(x, \xi)$ is the Green's function of the differential expression $\left(p u^{\prime}\right)^{\prime}+q u$ with boundary conditions (3.2). In discussing the last integral we restrict ourselves to the case $q \leqq 0$ in order that $R(u)$ be positive. For each couple of these three integrals it is possible to prove a pair of theorems concerning extrema.

The integrals $D(u), K_{0}(u), R(u)$ can be approximated as closely as we please by the corresponding integrals in which $u(x)$ possesses an absolutely continuous first derivative. Hence there is no loss of generality in restricting ourselves to the consideration of such functions $u$.

Theorems I-III concern the orthogonal case and IV the polar case.

THEOREM I. Among all continuous functions $u(x)$ which give to the integral $D(u)$ a meaning and which are subject to the condition $K_{0}=1$, the boundary conditions (3.2), and the infinity of linear conditions

$$
K_{i} \equiv \int_{0}^{1} k U_{i} u d x=0 \quad(i=1, \cdots, m-1 ; s+1, s+2, \cdots),
$$

the maximum value $\lambda_{8}$ of $D(u)$ is furnished by $U_{\text {s }}$ and the minimum value $\lambda_{m}$ is furnished by $U_{m}$. 
For, in the orthogonal case formulas analogous to (1.20), (1.21) have the simpler form

$$
K_{0}(u)=\sum_{1}^{\infty} c_{i}^{2}, \quad D(u)=\sum_{1}^{\infty} \lambda_{i} c_{i}^{2}
$$

and the hypotheses (3.3) reduce the problem to the consideration of relative extrema for quadratic forms in a finite number of variables only,

$$
D(u) \equiv \sum_{i=m}^{\dot{1}} \lambda_{i} c_{i}^{2}=\text { extremum, } \quad \sum_{i=m}^{\infty} c_{i}^{2}=1 .
$$

Since $\lambda_{\varepsilon}$ is the largest of the characteristic numbers here appearing, and $\lambda_{m}$ the smallest, the theorem is immediately established.

A consideration of the proof of Theorem I indicates that the reciprocal theorem can be at once deduced.

THEOREM Ia. Under the boundary and linear conditions of Theorem I and $q(x) \leqq 0$ the minimum of $K_{0}(u)$ for those values of $u$ which make $D(u)=1$ is $1 / \lambda_{\text {s }}$

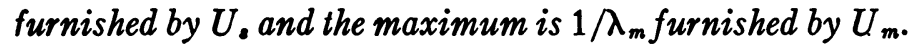

TheOREM II. Among all continuous functions $u(x)$ the integral $R(u)$, under the conditions $q \leqq 0, K_{0}=1$ and (3.2), (3.3), possesses a maximum value $1 / \lambda_{m}$ furnished by $U_{m}$ and a minimum value $1 / \lambda_{\text {s }}$ furnished by $U_{s}$.

For, on setting $h(x)=k(x) u(x)$ the formula (1.19) becomes

$$
R(u)=\sum_{i} \frac{\left(\int_{0}^{1} k(x) u(x) U_{i}(x) d x\right)^{2}}{\left|\lambda_{i}\right|}
$$

and from (1.20) and the hypotheses, this may be written

$$
R(u)=\sum_{m} \frac{c_{i}^{2}}{\lambda_{i}} .
$$

This with the second formula of (3.4), valid here also, is sufficient to establish the theorem.

TheOREM IIa. Under the boundary and linear conditions of Theorem II the maximum of $K_{0}$ for those values of $u$ which make $R(u)=1$ is $\lambda_{\text {s }}$ furnished by

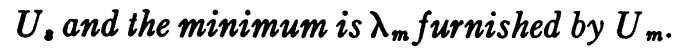

A consideration of the preceding theorems and of $(0.20)$ suggests another theorem which, with its reciprocal, may be readily proved by means of (3.4) and (3.5): 
Theorem III. Among all functions $u(x)$ which give $D(u)$ a meaning and are subject to the conditions that $R(u)=1, q(x) \leqq 0$, and (3.2), (3.3), the integral $D(u)$ possesses a minimum $\lambda_{m}^{2}$ furnished by $U_{m}$ and a maximum $\lambda_{s}^{2}$ furnished by $U_{\text {s. }}$

TheOREM IIIa. Under the boundary and linear conditions of Theorem III the maximum of the integral $R(u)$ subject to the condition $D(u)=1$ is $1 / \lambda_{m}{ }^{2}$ furnished by $U_{m}$ and the minimum is $1 / \lambda_{s}^{2}$ furnished by $U_{s .}$

In the polar case the situation allows only one extremum and the reciprocal theorem will have only one.

Theorem IV. Among all continuous functions $u(x)$ which give $D(u)$ a meaning and are subject to the conditions $K_{0}=1$, (3.2) and (3.3), the integral $D(u)$ possesses a minimum $\lambda_{m}$ furnished by $U_{m}$, while the maximum is infinite.

For as in Theorem I, by means of (1.20) and (1.21) and the hypothesis, the problem is reduced to relative extrema of quadratic forms with an infinite number of variables

$$
D(u)=\sum_{m}^{\dot{1}} \lambda_{i} c_{i}^{2}-\sum_{-\infty}^{-1} \lambda_{i} c_{i}^{2}=\text { extremum } ; \quad K_{0}=\sum_{m}^{\infty} c_{i}^{2}-\sum_{-\infty}^{-1} c_{i}^{2}=1
$$

On multiplication of the second of these by $\lambda_{m}$ and subtraction from the first, there results

$$
D(u)-\lambda_{m}=\sum_{m+1}^{\infty} c_{i}^{2}\left(\lambda_{i}-\lambda_{m}\right)+\sum_{-\infty}^{-1} c_{i}^{2}\left(\lambda_{m}-\lambda_{i}\right),
$$

and since all the coefficients of $c_{i}^{2}$ are positive, it is seen that the minimum is given by $c_{m}=1, c_{i}=0(i=\cdots,-2,-1 ; m+1, \cdots, s)$. On the other hand for $c_{m}=2^{1 / 2}, c_{-n}=1$, and the other $c$ 's zero the formulas (3.6) give $D(u)$ the value $2 \lambda_{m}-\lambda_{-n}$ and this may be made as great as is desired by taking $n$ large enough.

THeOREM IVa. Under the boundary and linear conditions of Theorem IV and provided $q \leqq 0$, the maximum $1 / \lambda_{m}$ of the integral $K_{0}$ for those values of $u$ which make $D(u)=1$ is furnished by $U_{m}$.

For, on multiplication of the second of the expressions

$$
K_{0}=\sum_{m}^{\infty} c_{i}^{2}-\sum_{-\infty}^{-1} c_{i}^{2}=\max ., D(u)=\sum_{m}^{\infty} \lambda_{i} c_{i}^{2}-\sum_{-\infty}^{-1} \lambda_{i} c_{i}^{2}=1
$$

by $1 / \lambda_{m}$ and subtraction from the first, it follows that 


$$
K_{0}-\frac{1}{\lambda_{m}}=\dot{\sum} c_{m+1}^{2}\left(1-\frac{\lambda_{i}}{\lambda_{m}}\right)+\sum_{-\infty}^{-1} c_{i}^{2}\left(\frac{\lambda_{i}}{\lambda_{m}}-1\right)
$$

and since all the coefficients of $c_{i}^{2}$ are negative the theorem follows at once.

If in Theorem IV, instead of setting $K_{0}$ equal to 1 it is equated to -1 and if in (3.3) the $U_{i}$ are replaced by $U_{-i}$ the minimum is $-\lambda_{m}$ furnished by $U_{-m}$. There is a corresponding reciprocal theorem.

\section{Generalization OF THE EXTREMUM PROBLEM.}

The EUler EQUATION AND ITS SOlUTIONS

If we generalize the problem (1.7), (1.8), (2.1) by seeking the minimum or maximum of

$$
D(u)=\int_{0}^{1}\left(p u^{\prime 2}-q u^{2}\right) d x ; \quad p>0, u(0)=u(1)=0,
$$

under the quadratic condition

$$
K_{0} \equiv \int_{0}^{1} k u^{2} d x=1,
$$

and the infinite number of linear conditions

$$
\begin{aligned}
K_{i} \equiv \int_{0}^{1} k U_{i} u d x & =0 \\
(i & =1, \cdots, m-1 ; s+1, s+2, \cdots ;(s \geqq m))
\end{aligned}
$$

the Lagrange method suggests the consideration of the absolute minimum of the integral

$$
\begin{aligned}
\int_{0}^{1}\left[p u^{\prime 2}-q u^{2}+\lambda\left(v_{0}^{\prime}-k u^{2}\right)\right. & +\sum_{1}^{m-1} 2 \mu_{i}\left(v_{i}^{\prime}-k U_{i} u\right) \\
& \left.+\sum_{i+1}^{\infty} 2 \mu_{i}\left(v_{i}^{\prime}-k U_{i} u\right)\right] d x,
\end{aligned}
$$

where after the analogy of (1.9) and (1.12) for the finite case, the $v$ 's are defined as follows:

(4.5) $v_{0}=\int_{0}^{x} k u^{2} d x, \quad v_{i}=\int_{0}^{x} k U_{i} u d x(i=1, \cdots, m-1 ; s+1, \cdots)$, which may also be written

$$
\begin{aligned}
& v_{0}^{\prime}-k u^{2}=0, \quad v_{0}(0)=0, \quad v_{0}(1)=1 ; \\
& v_{i}^{\prime}-k U_{i} u=0, \quad v_{i}(0)=v_{i}(1)=0 .
\end{aligned}
$$


It is natural to expect that the Euler equation will have a form

$$
\left(p u^{\prime}\right)^{\prime}+q u+\lambda k u+\sum_{1}^{m-1} \mu_{i} k U_{i}+\sum_{i+1}^{\infty} \mu_{i} k U_{i}=0
$$

generalized from (2.2) and the solutions

$$
u=\alpha u_{1}(x, \lambda)-\sum_{1}^{m-1} \frac{\mu_{i} U_{i}}{\lambda-\lambda_{i}}-\sum_{i+1}^{\infty} \frac{\mu_{i} U_{i}}{\lambda-\lambda_{i}}
$$

will be a generalized form of (2.3).

That the solutions (4.8) actually satisfy the equation (4.7) may be proved by direct substitution. To indicate the line of argument for deriving the Euler equation (4.7), we proceed formally and assume that $u_{1}(x)$ gives an extremum and set up admissible variations after the usual method. If the fundamental set of functions on which the variations are to be linearly dependent are chosen at random, the number of them must ordinarily be infinite. For, the family

$$
Y\left(x, \epsilon_{1}, \epsilon_{2}, \cdots\right)=u_{1}+\sum \epsilon_{j} \eta_{i}(k), \quad \eta_{j}(0)=\eta_{j}(1)=0
$$

is subject to a quadratic and an infinity of linear conditions and the $\epsilon$ 's must be chosen to satisfy them. If, however, it be noted that the linear conditions (4.3) are satisfied by any one of the functions* $U_{m}, \cdots, U_{\text {, }}$, or any linear combination of them, the problem is reduced to a much simpler one. For example,

$$
Y(x, \epsilon)=(1-\epsilon) U_{\iota}+\left(2 \epsilon-\epsilon^{2}\right)^{1 / 2} U_{l}, \quad m<l<s,
$$

satisfies not only the linear conditions but also the quadratic (4.2).

In the general case it is easily seen that the set (4.9) must satisfy the relations

$$
K_{0}=\int_{0}^{1} k\left[u_{1}+\sum_{i} \epsilon_{j} \eta_{j}(x)\right]^{2} d x=1, \quad K_{i}=\int_{0}^{1} k U_{i} \sum_{i} \epsilon_{i} \eta_{i} d x=0,
$$

and give to

$$
D\left(\epsilon_{1}, \epsilon_{2}, \cdots\right)=\int_{0}^{1}\left[p\left(u_{1}+\sum \epsilon_{j} \eta_{j}(x)\right)^{\prime 2}-q\left(u_{1}+\sum \epsilon_{j} \eta_{j}(x)^{2}\right]\right) d x
$$

an extremal value for $\epsilon_{i}=0$. It is then necessary that, for the values $\epsilon_{1}=\epsilon_{2}=\cdots=0$,

\footnotetext{
* In the polar case these functions are $\cdots U_{-2}, U_{-1}, U_{m}, \cdots, U$. The argument of this section is in general valid for that case also.
} 


$$
\begin{aligned}
\sum_{1}^{\infty} \frac{\partial D}{\partial \epsilon_{j}} d \epsilon_{j}=0, & \sum_{1}^{\infty} \frac{\partial K_{0}}{\partial \epsilon_{j}} d \epsilon_{j}=0, \quad \sum_{1}^{\infty} \frac{\partial K_{i}}{\partial \epsilon_{i}} d \epsilon_{j}=0 \\
& (i=1, \cdots, m-1, s+1, s+2, \cdots) ;
\end{aligned}
$$

hence whatever the multipliers $\lambda, \mu_{i}$ may be, it follows that

$$
d \epsilon_{1}\left(\frac{\partial M}{\partial \epsilon_{1}}\right)+\sum_{2}^{\infty} d \epsilon_{j}\left(\frac{\partial M}{\partial \epsilon_{j}}\right)=0
$$

where

$$
M=D-\lambda K_{0}-\sum_{1}^{m-1} 2 \mu_{i} K_{i}-\sum_{i+1}^{\infty} 2 \mu_{i} K_{i} .
$$

Let $\lambda, \mu_{i}$ be determined by the equations

$$
\frac{\partial M}{\partial \epsilon_{j}}=\frac{\partial D}{\partial \epsilon_{j}}-\lambda\left(\frac{\partial K_{0}}{\partial \epsilon_{j}}\right)-\sum_{1} \mu_{i}\left(\frac{\partial K_{0}}{\partial \epsilon_{j}}\right)=0 \quad(j=2,3, \cdots)
$$

which is possible provided the determinant of the coefficients of $\lambda$ and $\mu_{i}$

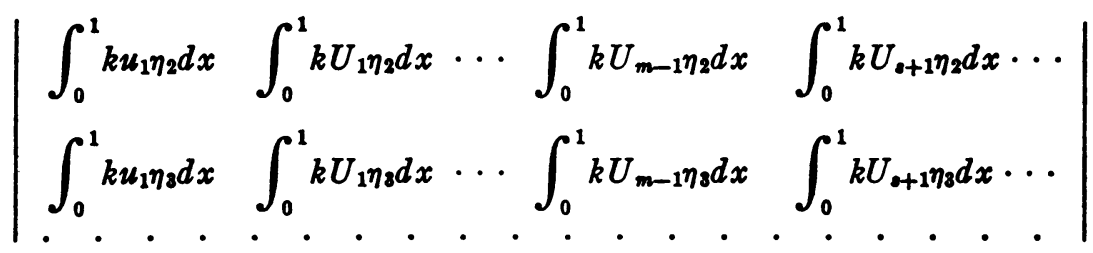

is different from zero. This may be ensured by proper choice of the $\eta$ 's; for example, diagonal terms may be made unity and all the other terms zero.

The values of $\lambda, \mu_{i}$ so chosen are independent of $\eta_{1}$. Hence from the formula $\partial M / \partial \epsilon_{1}=0$ derived by subtracting the infinite set of equations (4.11) from (4.10), the Euler equation (4.7) may be at once derived in the usual way.

Let us return to a discussion of the solutions (4.8) which may be regarded as an infinity-parameter set of plane extremals through the origin. Since the $U_{i}$ vanish at $x=1$, in order that $u$ vanish at that point also, it is necessary that $u_{1}(1, \lambda)=0$. For the minimizing or maximizing extremal of the family it may be shown that $\mu_{i}=0$ by the method used in deriving (1.15); in other words the extremum is a solution of the homogeneous equation (1.1). The function $u_{1}$ is then a solution of the homogeneous system (1.1), (1.2) and is orthogonal to $U_{i}$ unless it is a multiple of it. So far as we ascertain from the Euler equation, any one of the functions $U_{m}, \cdots, U$, corresponding to

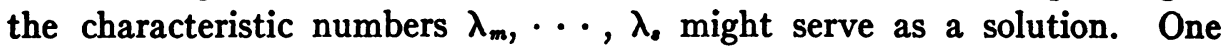
of these must give the minimum and one of them the maximum. 
To round out the discussion and prepare for the treatment of the Jacobi condition, the problem of extremals may be interpreted in infinity-dimension space $x u v_{0} v_{i}$. The Euler equations would in that case consist of (4.7), with the boundary conditions $u(0)=u(1)=0$, together with (4.6); the solutions constituting the infinity-parameter family of extremals through the origin would have a form generalized from (2.4) and would consist of (4.5) and (4.8).

In dealing with this family of extremals passing through the origin, it is natural to consider only those functions for which $\int_{0}^{1} k u^{2} d x$ is finite; an application of this condition to (4.8) shows that this limitation is equivalent to supposing that $\sum\left[\mu_{i} /\left(\lambda-\lambda_{i}\right)\right]^{2}$ is limited.

\section{THE SECOND VARIATION}

Despite the introduction of newer methods for the simple problem without auxiliary conditions, the method of second variation still remains standard for isoperimetric problems. It is then natural after the discussion of the Euler equation to proceed to the discussion of $\delta^{2} D$. For the admissible variations $\eta=\sum \epsilon_{i} \eta_{i}$ set up in (4.9) it is a necessary condition that, according as a minimum or maximum is sought, $\delta^{2} D \geqq 0$ or $\delta^{2} D \leqq 0$. Since by the nature of the hypotheses, the second variations $\delta^{2} K_{i}$ are 0 , this may also be written

$$
\delta^{2} D+\delta^{2} K_{0}+\sum_{1}^{m-1} \delta^{2} K_{i}+\sum_{i+1}^{\infty} \delta^{2} K_{i} \geqq 0 \text { or } \leqq 0 .
$$

On calculation from (4.4) it is found that

$$
\delta^{2} D=\epsilon^{2} \int_{0}^{1}\left(p \eta^{\prime 2}-q \eta^{2}-\bar{\lambda} k \eta^{2}\right) d x
$$

where $\bar{\lambda}$ is the characteristic number of the extremum solution and where $\eta$ is subject to the conditions

$$
\int_{0}^{1} k u \eta d x=0, \quad \int_{0}^{1} k U_{i \eta} d x=0 \quad(i=1, \cdots, m-1 ; s+1, \cdots) .
$$

By integrating (5.1) by parts and adding multiples of the linear terms (5.2) the second variation may be written

$$
\begin{gathered}
\delta^{2} D=-\epsilon^{2} \int_{0}^{1} \eta\left[\left(p \eta^{\prime}\right)^{\prime}+q \eta+\lambda k \eta+\alpha \delta \lambda k u+\sum_{1}^{m-1} \mu_{i} k U_{i}\right. \\
\left.+\sum_{i+1}^{\infty} \mu_{i} k U_{i}\right] d x
\end{gathered}
$$


In the orthogonal case* for the problem (4.1), (4.2), (4.3) the second variation related to the minimizing function $U_{m}$ is positive and that related to the maximizing function $U_{\text {, is negative. }}$

For, taking up first the problem of a maximum it is necessary that the integral

$$
\int_{0}^{1}\left(p \eta^{2}-q \eta^{2}-\lambda_{8} k \eta^{2}\right) d x
$$

be negative for all $\eta \neq 0$ satisfying the continuity and boundary conditions and the linear conditions (5.2); it will also satisfy a quadratic condition such as

$$
\int_{0}^{1} k \eta^{2} d x=c \neq 0 \text {. }
$$

The problem may be regarded as that of finding a maximum zero of (5.4) for those functions $\eta(x) \neq 0$. which satisfy (5.2) and (5.5). As Bliss has pointed out in similar problems, the original problem for the integral $D(u)$ may itself be put into precisely this form and the results there obtained applied here. The admissible variation $\eta$ must be linearly dependent on $U_{m}, \cdots$, $U_{.} ;$that is, $\eta=\sum_{i=m}^{s} a_{i} U_{i}$; on calculation it turns out in a manner analogous to Theorem I of $\$ 3$ that

$$
\delta^{2} D=\epsilon^{2} \sum_{m}^{\dot{1}}\left(\lambda_{i}-\lambda_{s}\right) a_{i}^{2} \leqq 0 .
$$

That $\delta^{2} D$ is actually negative may be seen by noting that it could be zero only if $a_{m}=\cdots=a_{s-1}=0$; since $\sum a_{i}^{2} \neq 0$, it follows that $a_{s} \neq 0$. But $U$, cannot be an admissible variation for $U_{\text {s }}$ itself since the value of $K_{0}$ would be affected; hence $\delta^{2} D<0$.

A similar argument shows that in the problem of a minimum the admissible variations of the functions $U_{m}$ make $\delta^{2} D$ positive.

From analogy with the Legendre condition for the finite problem, we would expect, in order that a maximum exist, that $H_{y^{\prime} y^{\prime}}=2 p$ (where $H$ is the integrand of (4.4)) must be negative while for a minimum this same function must be positive. But here we have found a maximum for $p>0$ in striking contradiction to the theorems for the finite problem. It is evident that there must be some underlying reason why one of these conditions and not the other is satisfied. As will be evident later, an investigation of

* For the minimum problem in the polar case (Theorem 4, \&3), it follows in similar fashion that

$$
\otimes D=\epsilon^{2} \sum_{m}^{\infty} a_{i}^{2}\left(\lambda_{i}-\lambda_{m}\right)+\epsilon^{2} \sum_{-\infty}^{-1} a_{i}^{2}\left(\lambda_{m}-\lambda_{i}\right)>0 \text {. }
$$


the Jacobi condition for the problem is fundamental before any appeal can be made to the Legendre condition.

It may be noted that all the admissible variations of the maximum problem are contained in the family of extremals of the minimum problem, while a part only of the admissible variations for the minimum problem are contained among the extremals of the maximum problem.

\section{THE JACOBI CONDITION FOR THE INFINITE PROBLEM}

In (1.3) there was set up a two-parameter family $\alpha u_{1}(x, \lambda)$ of solutions of the homogeneous equation (1.1) and in (4.8) an infinity-parameter family

$$
Y\left(x, \lambda, \mu_{i}\right) \equiv \alpha u_{1}(x, \lambda)-\sum_{1}^{m-1} \mu_{i} \frac{U_{i}}{\lambda-\lambda_{i}}-\sum_{i+1}^{\infty} \mu_{i} \frac{U_{i}}{\lambda-\lambda_{i}}
$$

of plane extremals which are solutions of the Euler equation (4.7) and which pass through the origin, the parameters $\mu_{i}$ being restricted to those values which make $\sum\left[\mu_{i} /\left(\lambda-\lambda_{i}\right)\right]^{2}$ finite. By means of the auxiliary variables $v_{0}(x), v_{1}(x), \cdots, v_{m-1}(x) ; v_{s+1}(x), \cdots$ as defined in (4.6), extremals were also set up in space of infinity dimensions $x u v_{0} v_{i}$. To every extremal (6.1) of the $x u$ space corresponds an extremal in the higher space. Among the questions which present themselves is that concerning the existence of a field in the neighborhood of the minimizing extremal in infinity dimensions. Does there exist a region about this curve through each point of which there passes a unique extremal of the family in infinity-dimensional space? In other words, do there exist constants $\alpha, \lambda, \mu_{i}$ such that for these values an extremal (6.1), (4.5) passes through the origin and any other designated point? Is there a one-to-one correspondence between the $x u v_{0} v_{i}$ space and the $\alpha \lambda \mu_{i}$ space? Or, on the contrary, is one extremal cut by a neighboring one before the end of the interval 0,1 is reached: that is, is the point conjugate to $x=0$ in the extended sense within the interval? The condition for a conjugate point has been developed in $\$ 2$ at considerable length for the finite problem and it is not necessary in extending it formally to the infinite problem that great detail be given.

For a conjugate point an infinity of conditions corresponding to (2.5) must be satisfied:

$$
\begin{gathered}
\alpha \delta \lambda \frac{\partial u_{1}}{\partial \lambda}+\delta \alpha u_{1}-\sum \delta \mu_{i} \frac{U_{i}}{\lambda-\lambda_{i}}=0 \\
2 \alpha^{2} \delta \lambda \int_{0}^{x} k u_{1} \frac{\partial u_{1}}{\partial \lambda} d x+2 \alpha \delta \alpha \int_{0}^{x} k u_{1}^{2} d x-2 \alpha \sum \frac{\delta \mu_{i}}{\lambda-\lambda_{i}} u_{1} U_{i} d x=0
\end{gathered}
$$


This leads to a consideration of the infinite determinant:

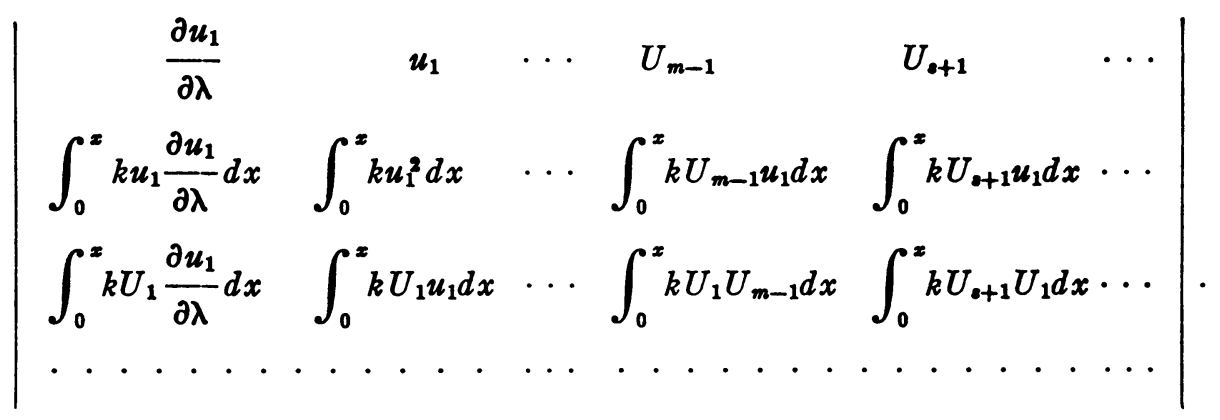

Denoting by $F_{m+1}(x), F_{m+2}(x), \cdots$ the principal $(m+1)$ th, $(m+2)$ th, ... order minors in its upper left-hand corner, it may easily be shown that the determinant (6.3), regarded as the limit of these minors, is a bounded function of $x$, being 0 at $x=0$ and 1 at $x=1$. From the previous paper* we have the theorems that each of the functions $F_{m+1}(x), F_{m+2}(x), \cdots$ are 0 at $x=0$ and positive elsewhere, being 1 at $x=1$, and that the quotient $F_{m+p} / F_{m}$ is, for all $p$, a monotone function ranging from 0 at $x=0$ to 1 at $x=1$. A passage to the limit gives a bounded function (6.3).

The formal analogon of the Jacobi condition may then be stated as follows: In order that there be no conjugate point in the interval, the infinite determinant (6.3) must have no zero other than $x=0$ in the interval.

To give a formal indication of the necessity of this condition let us assume that the equations (6.2) are satisfied for a point $x_{1}$ within the interval and prove that this involves the vanishing of $\delta^{2} D$. We have seen (5.3) that the second variation may be written

$$
\delta^{2} D=-\epsilon^{2} \int_{0}^{1} \eta\left[\left(p \eta^{\prime}\right)^{\prime}+q \eta+\lambda_{m} k \eta+\alpha \delta \lambda k u+\sum \mu_{i} k U_{i}\right] d x .
$$

Since for the minimizing extremal $\lambda=\lambda_{m}$ and $\mu_{i}=0$, it follows that $\mu_{i}=\delta \mu_{i}$, $\lambda-\lambda_{m}=\delta \lambda$; the expression in brackets in the integrand of (6.4) has then the form

$$
\left(p \eta^{\prime}\right)^{\prime}+q \eta+\lambda_{m} k \eta+\alpha \delta \lambda k u+\sum \delta \mu_{i} k U_{i} .
$$

It is readily shown that the substitution of the expression on the left of the first equation of (6.2) will make (6.5) zero. If then in the interval $0, x_{1}$ we

* Loc. cit., Mathematische Annalen, vol. 68, p. 289. 
choose for the variation $\eta$ this expression in (6.2) and in the sub-interval $x_{1}, 1$ set $\eta=0$, the second variation $\delta^{2} D$ vanishes.

That $\delta^{2} D$ can actually in that case be made negative can be shown by the following argument. Referring to the discussion of the second variation in $\$ 5$ it may be noted that were $\eta$ to furnish the minimum for the integral (5.4) under the conditions (5.2), (5.5), thus making the Euler equation of this subsidiary problem the same as that of the original extremum problem, the solution would have all its derivatives continuous at $x_{1}$, which is obviously not the case here. Hence the variation $\eta$ chosen above does not give a minimum to the second variation and $\delta^{2} D$ can be made negative. This would indicate that for a minimum the point conjugate to $x=0$ cannot be within the interval; and it indicates also that there must be a conjugate point in the interval if there is to be a maximum.

To consider the relation between the infinite determinants (6.3) for various values of $m$ and $s$ let us denote by $D_{14}$ the infinite determinant obtained by setting $m=1$ and $s=3$ and by $D_{134}$ that obtained by setting $m=1, s=2$. The latter contains one more row and column than the former and as is indicated in the Lemma in $\$ 2$, we have the formula

$$
\frac{d}{d x} \frac{D_{14}}{D_{134}}=\frac{\lambda_{2}-\lambda_{3}}{p D_{134}^{2}} \alpha_{14}^{2},
$$

where $\alpha_{14}$ is a certain first minor of $D_{134}$. In other words the discussion parallels exactly that of $\$ 2$ except that instead of a finite number of terms there is an infinite number. Each of the infinite determinants obtained by dropping out any finite number of columns and the corresponding rows (taken in order or scattered here and there throughout the determinant) can have no zero within the interval. By dropping out any column and corresponding row the zero of the determinant moves to the left. Since the ratio of any determinant to that of order lower by one is monotone in the interval 0,1 the same will be true concerning the ratio of any two in the scale provided the one is contained in the other.

7. Hamilton function. Hilbert integral. Weierstrass condition

Assuming that the Jacobi condition is satisfied in the interval 0, 1 , consider a point in the infinity-dimensional field about the maximizing or minimizing extremal. Through the origin and this point whose abscissa is $x$ there will be an extremal of the family

$$
u=\alpha u_{1}(x, \lambda)-\sum_{1}^{m-1} \frac{\mu_{1} U_{i}}{\lambda-\lambda_{i}}-\sum_{i+1}^{\infty} \frac{\mu_{i} U_{i}}{\lambda-\lambda_{i}},
$$




$$
v_{0}=\int_{0}^{x} k u^{2} d x, \quad v_{i}=\int_{0}^{x} k U_{i} u d x .
$$

The Hamilton function is defined to be the integral

$$
W\left(x, u, v_{0}, v_{1}, \cdots, v_{m-1}, v_{s+1}, \cdots\right)=\int_{0}^{x}\left(p u^{\prime 2}-q u^{2}\right) d x
$$

taken along this extremal. Since along this curve the relations (7.2) are satisfied, the integral may also be written

$$
\begin{aligned}
W=\int_{0}^{x}\left[p u^{\prime 2}-q u^{2}+\lambda\left(v_{0}^{\prime}-k u^{2}\right)\right. & +\sum_{1}^{m-1} \mu_{i}\left(v_{i}^{\prime}-k u U_{i}\right) \\
& +\sum_{i+1}^{\infty} \mu_{i}\left(v_{i}^{\prime}-k u U_{i}\right) d x .
\end{aligned}
$$

By the method usual in such cases* the derivatives may be calculated formally as follows:

$$
\begin{gathered}
\frac{\partial W}{\partial x}=p \varphi^{2}-q u^{2}-\varphi(2 p \varphi)-\sum_{1}^{m-1} \mu_{i} \varphi_{i}-\sum_{i+1}^{\infty} \mu_{i} \varphi_{i} ; \\
\frac{\partial W}{\partial u}=2 p \varphi ; \quad \frac{\partial W}{\partial v_{0}}=0 ; \quad \frac{\partial W}{\partial v_{i}}=\mu_{i},
\end{gathered}
$$

where $\phi$ is the slope of the projection on the $x u$ plane of the space extremal through the given point and $\varphi_{i}$ the slope of the projection of the space extremal on the $x v_{i}$ plane. Because of the linear character of the conditions, it follows from the definitions that both $\gamma_{i}$ and $\nu_{i}^{\prime}$ are equal to the value of $k u U_{i}$ at the point in question and hence are equal to one another. The differential $d W$ may then be written

$$
\begin{aligned}
d W=\left[-p p^{2}-q u^{2}\right. & \left.-\sum_{1}^{m-1} \mu_{i} v_{i}^{\prime}-\sum_{s+1}^{\infty} \mu_{i} v_{i}^{\prime}\right] d x+2 p q d u \\
& +\sum_{1}^{m-1} \mu_{i} d v_{i}+\sum_{i+1}^{\infty} \mu_{i} d v_{i}=-\left(p p^{2}+q u^{2}\right) d x+2 p p d u .
\end{aligned}
$$

Because this is a perfect differential its integral

$$
\int_{0}^{x}\left(-p p^{2}-q u^{2}+2 p p u^{\prime}\right) d x
$$

is independent of the path and is the analogon of the Hilbert independent integral for this problem.

* Bolza, p. 599. 
To set up the Weierstrass formula let us compare the value of $\left.D^{\prime \prime} u\right)$ taken for the interval 0,1 along a curve $C$ of admissible variation, which must satisfy the equations (7.2), with its value along the minimizing extremal. The integral (7.4) taken throughout the interval along the minimizing extremal is the Hamilton function and represents the minimum. Its value arong the admissible variation is the same. Hence

$$
\Delta J=\int_{C}\left[\left(p u^{\prime 2}-q u^{2}\right)+\left(p q^{2}+q u^{2}-2 p q u^{\prime}\right)\right] d x
$$

and on setting $E\left(x, u, u^{\prime}, \varphi\right)=p\left(u^{\prime}-\varphi\right)^{2}$ this may be written

$$
\Delta J=\int_{C} E\left(x, u, u^{\prime}, p\right) d x .
$$

The conditions $E\left(x, u, u^{\prime}, p\right) \geqq 0, E\left(x, u, u^{\prime}, p\right) \leqq 0$ would be the analogons of the Weierstrass conditions for minimum and maximum respectively in the finite problem.

Here $E\left(x, u, u^{\prime}, p\right) \geqq 0$ for both minimum and maximum, and the significance of this condition has entirely disappeared.

BroWN UNIVERSTTY,

Providencr, R. I. 